Physics

Physics Research Publications

\title{
Dimers on the triangular kagome lattice
}

Y. L. Loh

D. X. Yao

E. W. Carlson 


\title{
Dimers on the triangular kagome lattice
}

\begin{abstract}
Y. L. Loh, Dao-Xin Yao, and E. W. Carlson
Department of Physics, Purdue University, West Lafayette, Indiana 47907, USA

(Received 4 April 2008; revised manuscript received 1 September 2008; published 10 December 2008)

We derive exact results for close-packed dimers on the triangular kagome lattice (TKL), formed by inserting triangles into the triangles of the kagome lattice. Because the TKL is a nonbipartite lattice, dimer-dimer correlations are short ranged so that the ground state at the Rokhsar-Kivelson (RK) point of the corresponding quantum dimer model on the same lattice is a short-ranged spin liquid. Using the Pfaffian method, we derive an exact form for the free energy, and we find that the entropy is $\frac{1}{3} \ln 2$ per site, regardless of the weights of the bonds. The occupation probability of every bond is $\frac{1}{4}$ in the case of equal weights on every bond. Similar to the case of lattices formed by corner-sharing triangles (such as the kagome and squagome lattices), we find that the dimer-dimer correlation function is identically zero beyond a certain (short) distance. We find in addition that monomers are deconfined on the TKL, indicating that there is a short-ranged spin liquid phase at the RK point. We also find exact results for the ground-state energy of the classical Heisenberg model. The ground state can be ferromagnetic, ferrimagnetic, locally coplanar, or locally canted, depending on the couplings. From the dimer model and the classical spin model, we derive upper bounds on the ground-state energy of the quantum Heisenberg model on the TKL.
\end{abstract}

DOI: 10.1103/PhysRevB.78.224410

PACS number(s): 74.20.Mn, 75.10.Jm, 05.50.+q

\section{INTRODUCTION}

The nontrivial statistical mechanics problem of dimer coverings of lattices, which may be used to model, e.g., the adsorption of diatomic molecules onto a surface, ${ }^{1}$ experienced a renaissance with the discovery of exact mappings to Ising models. ${ }^{2,3} \mathrm{~A}$ second renaissance came with the search for ${ }^{4,5}$ and discovery of ${ }^{6}$ a true spin liquid phase with deconfined spinons. In the latter case, the problem of classical dimer coverings of a lattice illuminates the physics of the corresponding quantum dimer model. At the RokhsarKivelson (RK) point ${ }^{5}$ of the quantum dimer model, the ground states are an equal amplitude superposition of dimer coverings within the same topological sector, ${ }^{6,7}$ and in fact dimer correlations at this point correspond to the dimer correlations of the classical dimer model.

Results on classical hard-core dimer models in two ${ }^{3}$ and higher dimensions ${ }^{8}$ point to two classes of models, depending on the monomer-monomer correlation function, which is defined as the ratio of the number of configurations available with two test monomers inserted to the number of configurations available with no monomers present. On bipartite lattices (such as the square and honeycomb lattices), monomers are confined with power-law correlations. ${ }^{3,9}$ On nonbipartite lattices [such as the triangular, kagome, and the triangular kagome lattice (TKL) discussed here], monomers can be either confined or deconfined, and correlators exhibit exponential decay except at phase transitions. ${ }^{10-14}$ This implies that while the RK point of the quantum dimer model is critical on bipartite lattices, so that at $T=0$ a (critical) spin liquid exists only at the RK point, in nonbipartite lattices, such as the triangular lattice and lattices made of corner-sharing triangles such as the kagome and squagome lattices, it has been shown that the RK point corresponds to a disordered spin liquid. Correspondingly, it was established in both of these cases that there exist finite regions of parameter space where the ground state is a gapped spin liquid with deconfined spinons. Part of the interest in such states is the topological order that accompanies such ground states, and hence such states may be useful examples of the toric code. Interest also stems from the original proposals that the doped spin liquid phase leads to superconductivity. ${ }^{5,15}$

In this paper, we analyze the problem of classical closepacked dimers on the TKL, a nonbipartite lattice expected to display a spin liquid phase, as the first step in understanding the RK point of the corresponding quantum dimer model. The TKL, depicted in Fig. 1, has a physical analog in the positions of $\mathrm{Cu}$ atoms in the materials $\mathrm{Cu}_{9} X_{2}(\mathrm{cpa})_{6} \cdot x \mathrm{H}_{2} \mathrm{O}$ (cpa=2-carboxypentonic acid, a derivative of ascorbic acid;

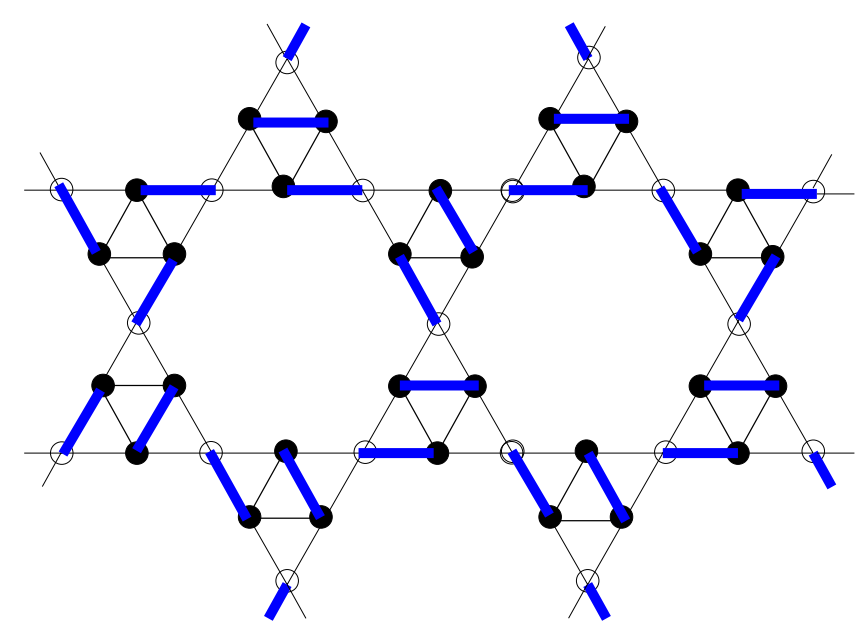

FIG. 1. (Color online) A dimer covering of a portion of the TKL. The TKL can be derived from the triangular lattice by periodically deleting seven out of every 16 lattice sites. This structure has two different sublattices $a$ (closed circles) and $b$ (open circles), which correspond to small trimers and large trimers, respectively. Each site has four nearest neighbors. The primitive unit cell contains $6 a$ sites, $3 b$ sites, $6 a$ - $a$ bonds, and $12 a$ - $b$ bonds. Thick lines represent dimers. A typical close-packed dimer covering is shown. 


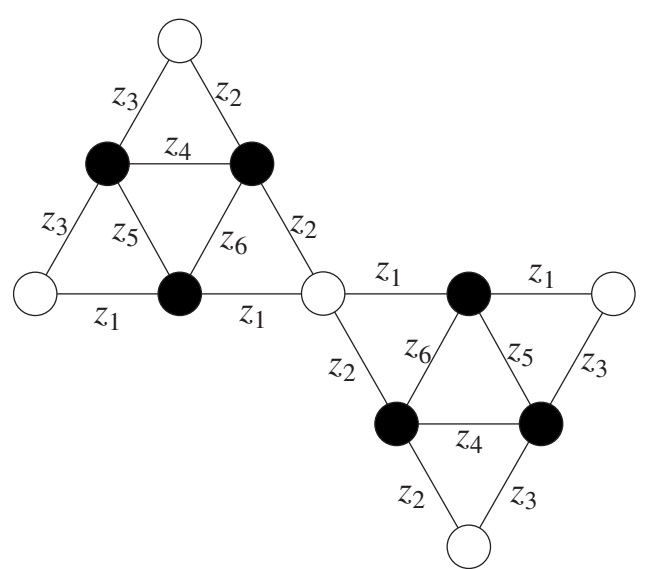

FIG. 2. Our assignment of weights $z_{\alpha}$ to bonds in the TKL. Solid (open) circles represent $a$ sites ( $b$ sites).

$X=\mathrm{F}, \mathrm{Cl}, \mathrm{Br}) .{ }^{16-18}$ We have previously studied Ising spins ${ }^{19}$ and $X X Z /$ Ising spins ${ }^{20}$ on the TKL; this paper represents an alternative approach to the problem. Using the well-known Pfaffian method, ${ }^{3}$ we obtain exact solutions of close-packed dimers on the TKL. We obtain an analytic form of the free energy for arbitrary bond weights. The entropy is $\frac{1}{3} \ln 2$ per site, independent of the weights of the bonds, $z_{a a}$ and $z_{a b}$. We find that the occupation probability of every bond is a constant $\frac{1}{4}$ in the absence of an orienting potential. The system has only local correlations, in that the dimer-dimer correlation function is exactly zero beyond two lattice constants, much like the situation on lattices made from corner-sharing triangles such as the kagome and squagome lattices. ${ }^{10} \mathrm{We}$ use exact methods to find the monomer-monomer correlation function and show that monomers are deconfined on the TKL. In addition, we solve for the ground states of the classical Heisenberg on this model. In addition to collinear phases (ferromagnetic and ferrimagnetic), we find a canted ferrimagnetic phase which interpolates smoothly between the two. We obtain a variational upper bound to the ground-state energy of the TKL quantum Heisenberg antiferromagnet using closed-packed dimer picture.

\section{MODEL, THERMODYNAMIC PROPERTIES, AND CORRELATION FUNCTION}

In this paper we consider the close-packed dimer model on the TKL, a lattice which can be obtained by inserting triangles inside of the triangles of the kagome lattice (see Fig. 1). The dimer generating function is defined as

$$
Z=\sum_{\text {dimer coverings }} \prod_{\langle i j\rangle} z_{i j}^{n_{i j}}
$$

where $\langle i j\rangle$ indicates a product over nearest-neighbor bonds, $z_{i j}$ is the weight on the bond joining site $i$ and site $j$, and $n_{i j}$ is the number of dimers (either 0 or 1 ) on bond $i j$ for the dimer covering under consideration. The term "closepacked" refers to the constraint that every lattice site must be occupied by one dimer, that is, those vacancies are not

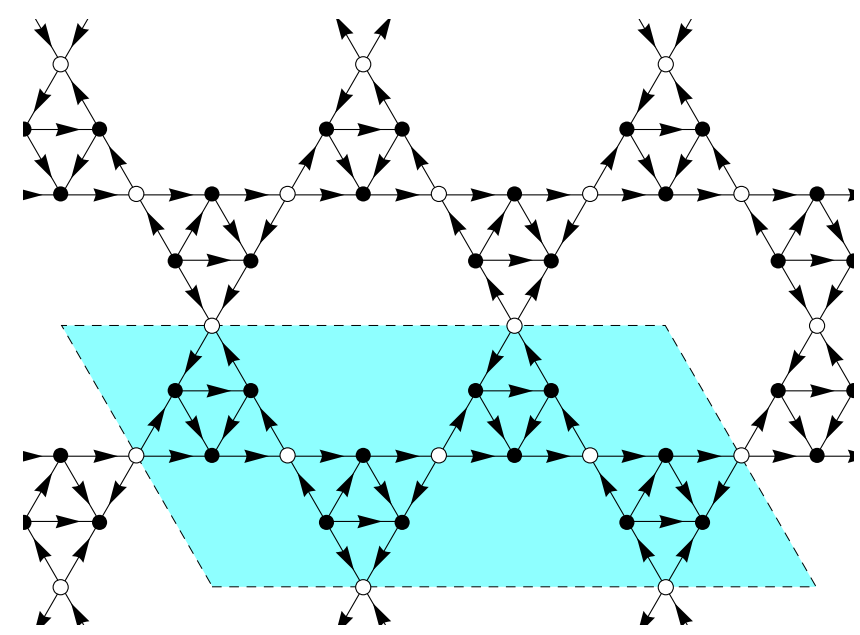

FIG. 3. (Color online) The arrows represent a Kasteleyn orientation (Ref. 3) on the TKL. Solid (open) circles represent $a(b)$ sublattices. The shaded region represents the doubled unit cell.

allowed. Therefore the number of sites $N_{\text {sites }}$ is twice the number of dimers $N_{\text {dimers }}=\Sigma_{\langle i j\rangle} n_{i j}$. We allow for the possibility different weights $z_{\alpha}=e^{-\beta \epsilon_{\alpha}}$ for six different types of bonds $\alpha=1,2,3,4,5,6$, as depicted in Fig. 2. Figure 1 shows an example of a dimer covering.

Several properties of this model, including the free energy, entropy, and dimer-dimer correlation function, can be calculated exactly using the well-known Pfaffian method. ${ }^{3}$ We begin by defining a Kasteleyn orientation ${ }^{3}$ (or Pfaffian orientation) for this lattice, i.e., a pattern of arrows laid on the bonds such that in going clockwise around any closed loop with an even number of bonds, there is an odd number of arrows pointing in the clockwise direction along the bonds. For the TKL, we have found that it necessary to double the unit cell in order to obtain a valid Kasteleyn orientation. ${ }^{21}$ Such an orientation is shown in Fig. 3. The doubled unit cell contains 18 sites.

The antisymmetric weighted adjacency matrix associated with this orientation, $A_{i j}$, is a $N_{\text {sites }} \times N_{\text {sites }}$ square matrix with a "doubly Toeplitz" block structure. The generating function of the dimer model is given by the Pfaffian of this matrix: $Z=\operatorname{Pf} A=\sqrt{\operatorname{det} A}$. In the infinite-size limit, this approaches an integral over the two-dimensional (2D) Brillouin zone,

$$
f=\lim _{N_{\text {sites }} \rightarrow \infty} \frac{F}{N_{\text {sites }}}=\frac{1}{18} \int_{0}^{2 \pi} \frac{d k_{x}}{2 \pi} \int_{0}^{2 \pi} \frac{d k_{y}}{2 \pi} \frac{1}{2} \ln \left|\operatorname{det} M\left(k_{x}, k_{y}\right)\right|,
$$

where we have normalized the free energy by the temperature such that $F \equiv \ln Z$ and where $M\left(k_{x}, k_{y}\right)$ is the $18 \times 18$ matrix below, 


$$
M=\left(\begin{array}{cccccccccccccccccc}
0 & z_{1} & z_{3} & 0 & 0 & 0 & 0 & 0 & 0 & 0 & 0 & 0 & 0 & 0 & 0 & 0 & \frac{z_{3}}{u} & -\frac{z_{1}}{u} \\
-z_{1} & 0 & -z_{5} & 0 & 0 & z_{1} & -z_{6} & 0 & 0 & 0 & 0 & 0 & 0 & 0 & 0 & 0 & 0 & 0 \\
-z_{3} & z_{5} & 0 & -v z_{3} & 0 & 0 & z_{4} & 0 & 0 & 0 & 0 & 0 & 0 & 0 & 0 & 0 & 0 & 0 \\
0 & 0 & \frac{z_{3}}{v} & 0 & -z_{2} & 0 & -\frac{z_{2}}{v} & -z_{3} & 0 & 0 & 0 & 0 & 0 & 0 & 0 & 0 & 0 & 0 \\
0 & 0 & 0 & z_{2} & 0 & z_{2} & 0 & z_{4} & z_{6} & 0 & 0 & 0 & 0 & 0 & 0 & 0 & 0 & 0 \\
0 & -z_{1} & 0 & 0 & -z_{2} & 0 & z_{2} & 0 & z_{1} & 0 & 0 & 0 & 0 & 0 & 0 & 0 & 0 & 0 \\
0 & z_{6} & -z_{4} & v z_{2} & 0 & -z_{2} & 0 & 0 & 0 & 0 & 0 & 0 & 0 & 0 & 0 & 0 & 0 & 0 \\
0 & 0 & 0 & z_{3} & -z_{4} & 0 & 0 & 0 & -z_{5} & -z_{3} & 0 & 0 & 0 & 0 & 0 & 0 & 0 & 0 \\
0 & 0 & 0 & 0 & -z_{6} & -z_{1} & 0 & z_{5} & 0 & z_{1} & 0 & 0 & 0 & 0 & 0 & 0 & 0 & 0 \\
0 & 0 & 0 & 0 & 0 & 0 & 0 & z_{3} & -z_{1} & 0 & z_{1} & z_{3} & 0 & 0 & 0 & 0 & 0 & 0 \\
0 & 0 & 0 & 0 & 0 & 0 & 0 & 0 & 0 & -z_{1} & 0 & -z_{5} & 0 & 0 & z_{1} & -z_{6} & 0 & 0 \\
0 & 0 & 0 & 0 & 0 & 0 & 0 & 0 & 0 & -z_{3} & z_{5} & 0 & -v z_{3} & 0 & 0 & z_{4} & 0 & 0 \\
0 & 0 & 0 & 0 & 0 & 0 & 0 & 0 & 0 & 0 & 0 & \underline{z_{3}} & 0 & z_{2} & 0 & -\frac{z_{2}}{v} & z_{3} & 0 \\
0 & 0 & 0 & 0 & 0 & 0 & 0 & 0 & 0 & 0 & 0 & 0 & -z_{2} & 0 & z_{2} & 0 & z_{4} & z_{6} \\
0 & 0 & 0 & 0 & 0 & 0 & 0 & 0 & 0 & 0 & -z_{1} & 0 & 0 & -z_{2} & 0 & z_{2} & 0 & z_{1} \\
0 & 0 & 0 & 0 & 0 & 0 & 0 & 0 & 0 & 0 & z_{6} & -z_{4} & v z_{2} & 0 & -z_{2} & 0 & 0 & 0 \\
-u z_{3} & 0 & 0 & 0 & 0 & 0 & 0 & 0 & 0 & 0 & 0 & 0 & -z_{3} & -z_{4} & 0 & 0 & 0 & -z_{5} \\
u z_{1} & 0 & 0 & 0 & 0 & 0 & 0 & 0 & 0 & 0 & 0 & 0 & 0 & -z_{6} & -z_{1} & 0 & z_{5} & 0
\end{array}\right),
$$

where, for brevity, we have written $u=e^{i k_{x}}$ and $v=e^{i k_{y}}$. The determinant of this matrix is independent of $k_{x}$ and $k_{y}$,

$$
\begin{aligned}
\operatorname{det} M\left(k_{x}, k_{y}\right)= & 64 z_{1}^{2} z_{2}^{2} z_{3}^{2}\left(z_{1} z_{4}+z_{2} z_{5}\right)^{2}\left(z_{1} z_{4}+z_{3} z_{6}\right)^{2} \\
& \times\left(z_{2} z_{5}+z_{3} z_{6}\right)^{2} .
\end{aligned}
$$

Taking the logarithm and integrating over the Brillouin zone gives the free energy per doubled unit cell. Hence, the free energy per site is

$$
f=\frac{1}{18} \ln \left[8 z_{1} z_{2} z_{3}\left(z_{1} z_{4}+z_{2} z_{5}\right)\left(z_{1} z_{4}+z_{3} z_{6}\right)\left(z_{2} z_{5}+z_{3} z_{6}\right)\right] .
$$

The occupation probability of each bond may be calculated by differentiating the free energy with respect to the weight of each bond. Let $N_{\alpha}$ be the total number of dimers on $z_{\alpha}$ bonds (as defined in Fig. 2), averaged over all configurations of the system. Since $Z=\Sigma_{\text {configs }} \Pi_{\alpha} z_{\alpha}^{N_{\alpha}}$, we have $N_{\alpha}=z_{\alpha} \frac{\partial F}{\partial z_{\alpha}}$. We define the occupation probability of each $\alpha$ bond as $p_{\alpha}=\frac{N_{\alpha}}{B_{\alpha}}$, where $B_{\alpha}$ is the total number of type- $\alpha$ bonds on the lattice. If $N_{\text {cells }}$ is the number of primitive unit cells, then $N_{\text {sites }}$ $=9 N_{\text {cells }}, B_{1}=B_{2}=B_{3}=4 N_{\text {cells }}$, and $B_{4}=B_{5}=B_{6}=2 N_{\text {cells. }}$. The results, normalized by the number of sites in the system, are

$$
p_{1}=\frac{1}{8}\left(1+\frac{z_{1} z_{4}}{z_{1} z_{4}+z_{3} z_{6}}+\frac{z_{1} z_{4}}{z_{1} z_{4}+z_{2} z_{5}}\right),
$$

$$
p_{4}=\frac{1}{4}\left(\frac{z_{1} z_{4}}{z_{1} z_{4}+z_{3} z_{6}}+\frac{z_{1} z_{4}}{z_{1} z_{4}+z_{2} z_{5}}\right) .
$$

Expressions for $p_{2}, p_{3}, p_{5}$, and $p_{6}$ follow by cyclic permutation of $\{1,2,3\}$ simultaneously with permutation of $\{4,5,6\}$. The entropy can be computed by the usual Legendre transformation, $S=F+\sum_{\alpha=1}^{6} \beta \epsilon_{\alpha} N_{\alpha}{ }^{22}$

The behavior of the correlation functions can be deduced in the same way as in Ref. 10. To find the dimer-dimer correlation functions, the standard method is to first calculate the "fermion" Green's function, which is the inverse of matrix $A$, Fourier transform it to real space, and use the result to construct the dimer-dimer correlation functions. The inverse of matrix $A, G\left(k_{x}, k_{y}\right)=\left[A\left(k_{x}, k_{y}\right)\right]^{-1}$, can be written as the matrix of cofactors of $A$ divided by the determinant of $A$. Since $\operatorname{det} A$ is independent of $k_{x}$ and $k_{y}$, the only dependence on $k_{x}$ and $k_{y}$ enters through the cofactor matrix. Each cofactor is at most a monomial in $e^{i k_{x}}$ and $e^{i k_{y}}$. From the rules of Fourier transformation it is easily seen that the real-space Green's function $G(x, y)$ is zero when $|x|>1$ or $|y|>1$ is greater than a certain cutoff distance. Hence the dimer-dimer correlation function will be zero beyond a distance of two unit cells. This is true regardless of the values of the bond weights depicted in Fig. 2. This extremely short-ranged behavior of the correlation function is similar to that for dimers on the kagome lattice ${ }^{10}$ and also to the spin-spin correlation for Ising spins in the frustrated parameter regime. ${ }^{19}$ It under- 
scores the special role played by kagomelike lattices (cf. Refs. 10 and 23).

Whereas quantum dimer models on bipartite lattices do not support deconfined spinons, quantum dimer models on nonbipartite lattices can have deconfined spinons. The connection to classical dimer models is that at the RK point, correlations in the quantum dimer model are the same as the correlations of the corresponding classical dimer problem. The only nonbipartite lattice for which deconfined spinons have been rigorously demonstrated is the triangular lattice by explicitly calculating the classical monomer-monomer correlation function using Pfaffian methods. ${ }^{11}$ On the kagome lattice, while no correspondingly rigorous calculation of the monomer-monomer correlation function has yet been demonstrated, there have been several indications that the spinons in quantum dimer models on the kagome lattice are deconfined (and, therefore, classical monomer-monomer correlators are similarly deconfined) from, e.g., the energetics of static spinon configurations, ${ }^{10}$ the behavior of the single-hole spectral function $^{24}$ and in the limit of easy-axis anisotropy. ${ }^{25}$ We have calculated the monomer-monomer correlation for the kagome lattice dimer model using the Pfaffian approach of Fisher and Stephenson, ${ }^{9}$ and we find that it is strictly constant, with $M(r)=1 / 4$ for any $r>0 .{ }^{26}$ Because the triangular kagome lattice dimer model maps to the kagome dimer model (with an extra degeneracy of four per unit cell), the monomer-monomer correlation on the TKL is also $M(r)$ $=1 / 4$ for monomers on any two $b$ sites or for any combination of $a$ and $b$ sites at least three sites apart.

\section{EFFECTS OF AN ORIENTING POTENTIAL}

In the $\mathrm{Cu}_{9} X_{2}(\mathrm{cpa})_{6} \cdot x \mathrm{H}_{2} \mathrm{O}$ materials, ${ }^{16-18}$ the $a$ spins are closer to each other than they are to the $b$ spins so the exchange couplings satisfy $\left|J_{a a}\right|>\left|J_{a b}\right|$. In the classical dimer approximation described in Sec. V, this corresponds to unequal weights for dimers on $a b$ bonds vs those on $a a$ bonds, $\left|z_{a a}\right|>\left|z_{a b}\right|$. Aside from this intrinsic difference in bond weights, it may also be possible to apply anisotropic mechanical strain to vary the lattice geometry (and, hence, the exchange couplings and dimer weights) in different directions.

To obtain some insight into the behavior of the classical dimer model under these conditions, we write $z_{\alpha}=e^{-\beta \epsilon_{\alpha}}$, where $\beta=1 / T$ is the inverse temperature and $\epsilon_{\alpha}$ is the potential energy for dimers on bond $\alpha$. We use the following parametrization for the potential energy on each site:

$$
\begin{aligned}
& \epsilon_{1}=\epsilon_{a b}-\delta, \quad \epsilon_{2}=\epsilon_{3}=\epsilon_{a b}, \\
& \epsilon_{4}=\epsilon_{a a}-\delta, \quad \epsilon_{5}=\epsilon_{6}=\epsilon_{a a},
\end{aligned}
$$

where $\delta$ is an orienting potential (i.e., an anisotropy parameter) which favors dimers in one direction. The bond occupation probabilities and entropy are independent of the values of $\epsilon_{a b}$ and $\epsilon_{a a}$ and depend smoothly on $\beta \delta$ (see Fig. 4),

$$
p_{1}=\frac{1}{8}(2+\tanh \beta \delta)
$$

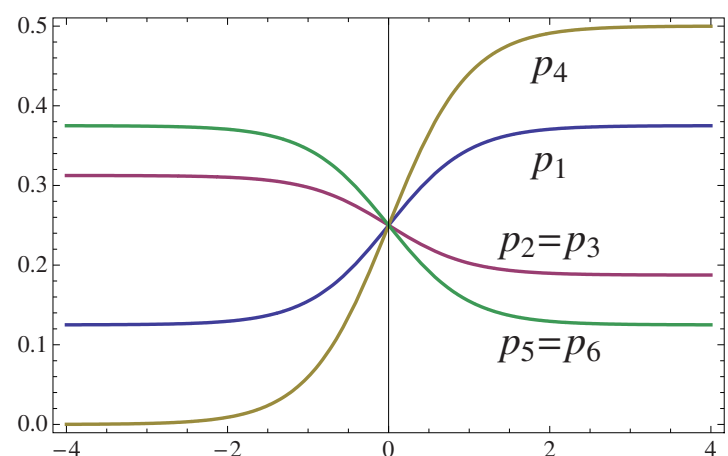

(a)

$\beta \delta$

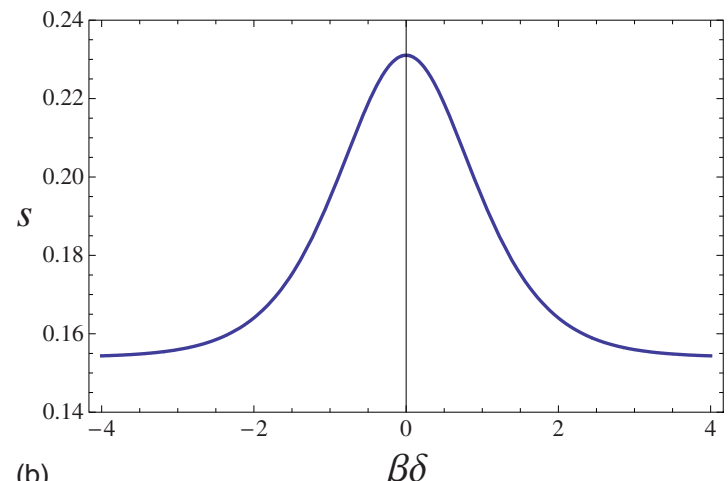

(b)

$\beta \delta$

FIG. 4. (Color online) Bond occupation probabilities and entropy per site as functions of the orienting field $\beta \delta$ defined in the text.

$$
\begin{gathered}
p_{4}=\frac{1}{4}(1+\tanh \beta \delta), \\
p_{2}=p_{3}=\frac{1}{16}(4-\tanh \beta \delta), \\
p_{5}=p_{6}=\frac{1}{8}(2-\tanh \beta \delta), \\
s=\frac{S}{N_{\text {sites }}}=\frac{1}{18}\left[\ln \left(64 \cosh ^{2} \beta \delta\right)-2 \beta \delta \tanh \beta \delta\right] .
\end{gathered}
$$

These results show that the TKL dimer model has neither a deconfinement transition (as a function of $\epsilon_{a b}-\epsilon_{a a}$ ) nor a Kasteleyn transition ${ }^{3}$ (as a function of $\delta$ ). It does, however, have a Curie-type "polarizability" with respect to an orienting potential. This is in contrast to the situation on the kagome lattice, ${ }^{23}$ where the bond occupation probabilities do not depend on the orienting potential.

\section{RESULTS FOR SYMMETRICAL CASE}

In the absence of the orienting potential (i.e., $\delta=0$ ), the expressions for the bond occupation probabilities and entropy become very simple,

$$
p_{\alpha}=\frac{1}{4}, \quad \alpha=1,2,3,4,5,6,
$$


TABLE I. Properties of close-packed dimer models on various lattices. Entropies are quoted per site. "Local" means that the correlation function is exactly zero beyond a certain radius-it has "finite support." The triangular, kagome, and triangular kagome lattices have deconfined monomers. The honeycomb dimer model not only has a finite dimer polarizability, but it has a Kasteleyn transition (Ref. 3) at $\delta=\delta_{c}$. The polarizability describes the changes in bond occupation probabilities induced by an orienting potential $\delta$.

\begin{tabular}{lcccc}
\hline \hline Lattice & Entropy & Dimer correlation & Monomer correlation & Polarizability \\
\hline Square $^{\mathrm{a}}$ & 0.2915609 & $r^{-2}$ & $r^{-1 / 2}$ & Finite \\
Honeycomb $^{\mathrm{b}}$ & 0.161533 & $r^{-2}$ & $r^{-1 / 2}$ & Kasteleyn transition \\
Triangular $^{\mathrm{c}}$ & 0.4286 & $e^{-r / 0.6014}$ & const $+e^{-r / 0.6014}$ & Finite \\
Kagome $^{\mathrm{e}}$, & $\frac{1}{3} \ln 2=0.231049$ & Local & Deconfined & 0 \\
TKL & $\frac{1}{3} \ln 2=0.231049$ & Local & Deconfined & Finite \\
\hline \hline
\end{tabular}

aReference 9 .

${ }^{\mathrm{b}}$ Reference 27.

${ }^{\mathrm{c}}$ Reference 11.

${ }^{\mathrm{d}}$ Reference 10 .

${ }^{\mathrm{e}}$ Reference 23 .

$$
s=\frac{1}{3} \ln 2 .
$$

Note that these quantities are independent of the relative bond weights $z_{a a}$ and $z_{a b}$. The comparison with other lattices in Table I shows that the entropy per site for the TKL is the same as that for the kagome lattice. Although the two lattices are related, this is, in fact, a coincidence for the following reason. The similarity can be seen by considering the number of $b$ spins per unit cell, which have a dimer that connects to a different unit cell. Because there is an odd number of sites per unit cell, this number must be odd, i.e., either one or three. Since the $b$ spins themselves form a kagome lattice, the same is, in fact, true of the kagome lattice. The difference is that for a given pattern of external dimers connecting to $b$ spins, there is no further degeneracy in the kagome case, whereas for the TKL there are four different internal dimer patterns corresponding to any given pattern of external dimers connecting to the $b$ spins. This means that the TKL has a further fourfold degeneracy so that the kagome entropy per unit cell of $s_{\text {cell }}=\ln 2$ becomes an entropy per unit cell of $s_{\text {cell }}=\ln 8=3 \ln 2$ in the TKL. Since there are nine spins per unit cell in the TKL, this yields $s=(1 / 3) \ln 2$ per site.

The total numbers of dimers on $a-a$ bonds and on $b-b$ bonds are

$$
\begin{aligned}
& N_{a a}=\frac{1}{3} N_{\text {dimers }}, \\
& N_{a b}=\frac{2}{3} N_{\text {dimers }},
\end{aligned}
$$

where $N_{\text {dimers }}$ is the total number of dimers and $N_{\text {dimers }}$ $=\frac{1}{2} N_{\text {sites }}$. (Of course, $N_{a a}=N_{4}+N_{5}+N_{6} \quad$ and $N_{a b}=N_{1}+N_{2}+N_{3}$.) Note that because there are twice as many $a-b$ bonds in the lattice as there are $a-a$ bonds, this implies that the dimer density is the same on every bond, regardless of the weights of the bonds. Since the number of sites is twice the number of dimers in the close-packed case, $N_{\text {sites }}$ $=2 N_{\text {dimers }}$, there are on average 9/2 dimers per unit cell. One third of those are on the $a-a$ bonds or $3 / 2$ per unit cell. Since there are six $a$ - $a$ bonds per cell, there are $(3 / 2) / 6=1 / 4$ dimers per $a-a$ bond. A similar analysis shows that there are $1 / 4$ dimers per $a$ - $b$ bond. In other words, there are $1 / 4$ dimers per bond, regardless of the relative weights $z_{a a}$ and $z_{a b}$ and regardless of whether it is an $a-a$ or $a-b$ bond. Under the constraint of close packing, the dimer densities are set by geometry rather than by energetics, similar to case of classical dimers on the kagome lattice. ${ }^{23,28-30}$

Our results for close-packed classical dimers on the TKL are summarized in Table I along with known results for the corresponding properties on the square, honeycomb, triangular, and kagome lattices. Notice that the kagome and TKL are special in having simple closed-form expressions for the entropies. In fact, the entropy per unit cell in each case is the logarithm of an integer. On triangular lattice as well as on the two bipartite lattices which are shown in the table (square and honeycomb), the entropy is not expressible as the logarithm of an integer.

The square and honeycomb lattices, being bipartite, admit a mapping to a solid-on-solid model ${ }^{31}$ and therefore have power-law correlations for both the dimer-dimer correlations and the monomer-monomer correlations. In the corresponding quantum dimer models, these lattices do not support deconfined spinons. As conjectured in Ref. 11, the nonbipartite lattices have exponential (or faster) falloff of the dimerdimer correlations. In the triangular, kagome, and TKL lattices, monomers are deconfined, which means that spinons are deconfined in the corresponding quantum dimer model at the RK point. In fact, Moessner and Sondhi ${ }^{6}$ showed that there is a finite region of parameter space in which a stable spin liquid phase is present on the triangular lattice.

\section{BOUNDS ON THE GROUND-STATE ENERGY OF THE QUANTUM HEISENBERG MODEL}

It is thought that the materials $\mathrm{Cu}_{9} X_{2}(\mathrm{cpa})_{6} \cdot x \mathrm{H}_{2} \mathrm{O}$ can be described in terms of quantum $S=1 / 2$ spins on the $\mathrm{Cu}$ atoms coupled by superexchange interactions. Nearest-neighbor 
isotropic antiferromagnetic couplings between $S=1 / 2$ spins on a 2D lattice with sublattice structure can lead to Néel order. For example, two-sublattice Néel order is favored on the square lattice, whereas three-sublattice Néel order is favored on the triangular lattice. ${ }^{32}$ However, on the kagome lattice and the TKL, quantum fluctuations are much more severe, and there is a possibility that they may lead to alternative ground states (such as valence-bond liquids).

A valence-bond state is a direct product of singlet pair states. Using a fermionic representation for the spins,

$$
\left.\left|\Psi_{\{n\}}\right\rangle=\left[\prod_{\langle i j\rangle} \frac{1}{\sqrt{2}}\left(c_{i \uparrow}^{\dagger} c_{j \downarrow}^{\dagger}-c_{i \downarrow}^{\dagger} c_{j \uparrow}^{\dagger}\right)^{n_{i j}}\right] \mid \text { vacuum }\right\rangle,
$$

where $n_{i j}=0$ or 1 is the number of valence bonds on bond $i j$, just as in Eq. (1).

Consider a quantum Hamiltonian with isotropic antiferromagnetic Heisenberg interactions,

$$
\hat{H}=-\sum_{\langle i j\rangle} J_{i j} \hat{\mathbf{S}}_{i} \cdot \hat{\mathbf{S}}_{j},
$$

where $J_{i j}$ is negative. The expectation value of this Hamiltonian in a valence-bond state is

$$
\left\langle\Psi_{\{n\}}\left|J_{i j} \hat{\mathbf{S}}_{i} \cdot \hat{\mathbf{S}}_{j}\right| \Psi_{\{n\}}\right\rangle=-\frac{3}{4} \sum_{\langle i j\rangle} n_{i j}\left|J_{i j}\right| .
$$

For close-packed dimers, the densities of valence bonds on $a-a$ and $a-b$ bonds are given by Eqs. (17) and (18). Therefore, the total energy of the close-packed valence-bond "trial wave function" is

$$
\begin{aligned}
E_{\mathrm{VB}} & =-\frac{3}{4}\left(N_{a a}\left|J_{a a}\right|+N_{a b}\left|J_{a b}\right|\right) \\
& =-\frac{1}{4}\left(\left|J_{a a}\right|+2\left|J_{a b}\right|\right) N_{\text {dimers }} \\
& =-\frac{1}{8}\left(\left|J_{a a}\right|+2\left|J_{a b}\right|\right) N_{\text {sites }} .
\end{aligned}
$$

This serves as an upper bound of the ground-state energy of the quantum Heisenberg model. Of course, matrix elements of the Hamiltonian which connect one dimer covering to another can serve to lower the actual energy even further.

One may also consider a more dilute dimer state. For large $\left|J_{a a}\right|$, one may expect dimers to preferentially occupy $a-a$ bonds so that hexamers with three $a-b$ bonds are disallowed. In such a trial dimer state, the associated energy is

$$
E_{\text {dilute }}=-\frac{1}{6}\left(\left|J_{a a}\right|+\left|J_{a b}\right|\right) N_{\text {sites }} .
$$

As shown in Fig. 5, this upper bound to the ground-state energy is lower than the others for large $\left|J_{a a}\right|$. If $J_{a b}$ is ferromagnetic and $J_{a a}$ is still antiferromagnetic, we expect another diluted dimer state, where dimers preferentially occupy $a-a$

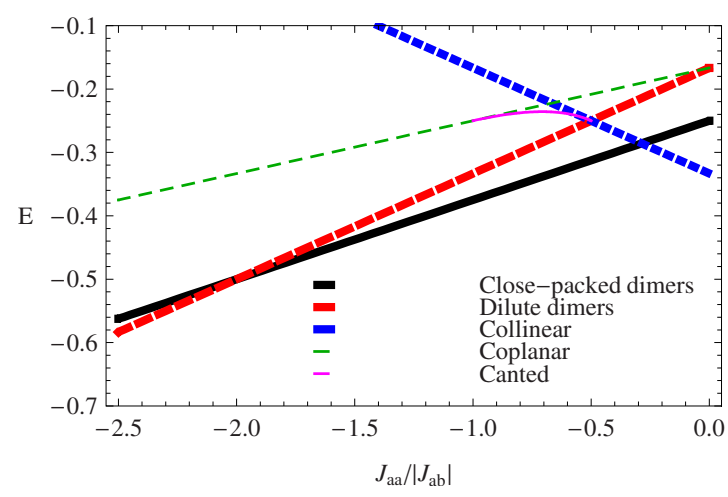

FIG. 5. (Color online) Comparison of upper bounds on the ground-state energy per site of the quantum Heisenberg model on the TKL, obtained by considering various trial wave functions. In the figure, we have set $S=1 / 2$.

bonds and other spins tend to be aligned (ferromagnetic phase). The corresponding energy is

$$
E_{\text {dilute+FM }}=-\left(\frac{1}{6}\left|J_{a a}\right|+\frac{1}{9}\left|J_{a b}\right|\right) N_{\text {sites }} .
$$

Other bounds can be obtained by considering the classical ground states of the Heisenberg model on the TKL (in which the spins are three vectors of magnitude $S=1 / 2$ ). In the materials of interest, there is not yet consensus whether the coupling $J_{a b}$ is ferromagnetic or antiferromagnetic. However, the Hamiltonian of the classical Heisenberg model is invariant under the transformation $S_{b} \rightarrow-S_{b}$ with $J_{a b} \rightarrow-J_{a b}$ so the thermodynamics are independent of the sign of $J_{a b}$.

First, let us consider classical Heisenberg spins on a single hexamer. By direct minimization of the energy of a single hexamer, we find that its classical ground state may be collinear, coplanar, or canted. For $J_{a a}>-\left|J_{a b}\right| / 2$, the ground state is collinear; the $a$ spins are aligned with each other, the $b$ spins are aligned with each other, and the $a$ and $b$ spins are parallel if $J_{a b}$ is ferromagnetic or antiparallel if $J_{a b}$ is antiferromagnetic. For $J_{a a}<-\left|J_{a b}\right|$, the ground state is coplanar; the $a$ spins are at $120^{\circ}$ to each other, the $b$ spins are at $120^{\circ}$ to each other, and adjacent $a$ and $b$ spins are at $60^{\circ}$ if $J_{a b}$ is ferromagnetic or at $120^{\circ}$ if $J_{a b}$ is antiferromagnetic. At intermediate couplings, $-\left|J_{a b}\right|<J_{a a}<-\left|J_{a b}\right| / 2$, the ground state is a canted state in which neither the $a$ spins nor the $b$ spins are coplanar; rather, each sublattice is canted away from Néel order, and each sublattice is canted away from the other. We define the canting angles of the $a$ and $b$ spins, $\alpha$ and $\beta$, such that $\alpha=\beta=0$ in the collinear state (see Fig. 6). The canting angles evolve continuously from $0^{\circ}$ (collinear) to $90^{\circ}$ (coplanar) as a function of the coupling ratio $J_{a a} /\left|J_{a b}\right|$ (see Fig. 7): the classical ground state has two continuous transitions.

Now, we observe that each of these hexamer states can tile the kagome lattice. Therefore, the ground-state energy of each hexamer can be used to deduce the ground-state energy of the entire system. In the collinear regime $\left(J_{a a}>-\left|J_{a b}\right| / 2\right)$, the collinear hexamer states lead to a unique global spin configuration [up to a global $\mathrm{SU}(2)$ rotation], so there is long-range ferromagnetic order (if $J_{a b}>0$ ) or ferrimagnetic order (if $J_{a b}<0$ ), and there is no macroscopic residual en- 


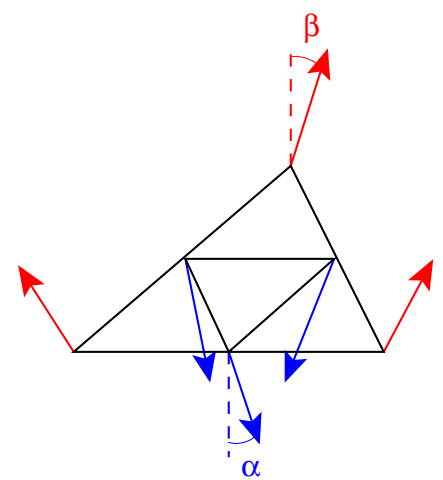

FIG. 6. (Color online) Canted state of a hexamer of classical Heisenberg spins on the TKL. $\alpha$ and $\beta$ are the canting angles of the $a$ and $b$ spins from the vertical axis. When $\alpha=\beta=0$, this reduces to a collinear state (which is ferromagnetic or antiferromagnetic depending on the sign of $J_{a-b}$ ). When $\alpha=\beta=\pi / 2$, it reduces instead to a coplanar state, in which the spins are all at $\pi / 3$ to each other.

tropy. The ground-state energy of the system is

$$
E_{\text {collinear }}=\frac{1}{6}\left(\left|J_{a a}\right|-2\left|J_{a b}\right|\right) N_{\text {sites }} .
$$

In the coplanar regime $\left(J_{a a}<-\left|J_{a b}\right|\right)$, there are infinitely many ways to tile the TKL with coplanar hexamer configurations (e.g., corresponding to three-sublattice or ninesublattice Néel order). Furthermore, there are an infinite number of zero modes (rotations of a few spins that cost zero energy). The ground-state energy is

$$
E_{\text {coplanar }}=-\frac{1}{12}\left(\left|J_{a a}\right|+2\left|J_{a b}\right|\right) N_{\text {sites }} .
$$

The physics is essentially the same as that of the classical Heisenberg kagome model. For that model, the prevailing point of view ${ }^{33-36}$ is that globally coplanar configurations are selected at finite temperature via an order-by-disorder mechanism, and the spin chiralities develop nematic order; recently, Zhitomirsky ${ }^{37}$ argued that there is an additional octupolar ordering, which is, in fact, the true symmetrybreaking order parameter.

The canted regime $-\left|J_{a b}\right|<J_{a a}<-\left|J_{a b}\right| / 2$ has the interesting property that, in general, $\alpha \neq \beta$, so there is a net magnetic moment on each hexamer. We have found that there are still infinitely many ways to tile the TKL and that there are still an infinite number of zero modes. It is possible that the zero modes cause the directions of the local moment to vary from place to place, destroying the long-range order with net magnetization; however, it is conceivable that the spincorrelation length gradually increases toward infinity in going from the locally coplanar state to the collinear state. The energy of the canted state is

$$
\begin{aligned}
E_{\text {canted }}= & \frac{2}{9}\left(-\frac{7\left|J_{a a}\right|}{4}+\frac{5 J_{a b}^{2}}{8\left|J_{a a}\right|}\right. \\
& \left.-\left|J_{a b}\right| \sqrt{\left(1-J_{a a}^{2} / J_{a b}^{2}\right)\left(J_{a b}^{2} / J_{a a}^{2}-1\right)}\right) N_{\text {sites }} .
\end{aligned}
$$

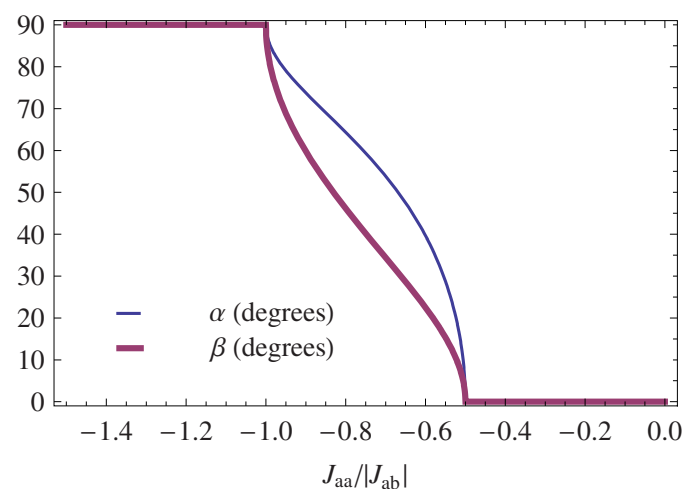

FIG. 7. (Color online) Canting angles in the ground state of the classical Heisenberg model on the TKL for as a function of coupling ratio $J_{a-a} /\left|J_{a-b}\right|$. The thin line shows the canting angle $\alpha$ of the $a$ spins and the thick line shows the canting angle $\beta$ of the $b$ spins, with respect to the collinear state, which is ferromagnetic or antiferromagnetic depending on the sign of $J_{a-b}$.

Equations (27)-(29) are the exact ground-state energies for the classical Heisenberg model on the TKL. They serve as upper bounds on the ground-state energy for the quantum Heisenberg model. Figure 5 shows these upper bounds, plotted together with the upper bounds derived from dimer coverings [Eqs. (24) and (25)], as explained earlier in this section. Notice that the upper bound for the ground-state energy set by considering dimer configurations beats the classical ground states for $J_{a a}$ large and negative (antiferromagnetic). In this highly frustrated regime, we expect that the true ground state of the quantum Heisenberg model is significantly modified by quantum fluctuations from that of the classical case.

\section{CONCLUSIONS}

In conclusion, we have studied the close-packed dimer model on the triangular kagome lattice (TKL) using exact analytic methods. We find that (in the absence of an orienting potential) the entropy is $s=\frac{1}{3} \ln 2$ per site, regardless of the weights of the bonds, $z_{a a}$ and $z_{a b}$. The occupation probability of every bond is $p_{\alpha}=\frac{1}{4}$. The dimer-dimer correlation function vanishes identically beyond two lattice sites, faster than that in the triangular lattice and similar to the falloff in the case of the kagome lattice. ${ }^{10}$ The monomer-monomer correlation function is $M(r)=1 / 4$ for $r$ greater than two lattice constants, indicating that monomers are deconfined in this lattice. This implies that the Rokhsar-Kivelson point ${ }^{5}$ of the corresponding quantum dimer model is a short-ranged deconfined spin liquid.

In addition, we find that the classical ground state of the Heisenberg model on the TKL is ferromagnetic (if $J_{a b}$ is ferromagnetic) or ferrimagnetic (if $J_{a b}$ is antiferromagnetic) when the coupling between $a$ spins on small trimers is large enough compared to the coupling between $a$ spins and $b$ spins, $J_{a a}>-\left|J_{a b}\right| / 2$. For $J_{a a}<-\left|J_{a b}\right|$, the ground state of a single hexamer is a coplanar state, and the physics reduces to that of the classical Heisenberg kagome model..$^{33-36}$ In between, there is a canted classical ground state in which the $a$ 
spins and $b$ spins within a hexamer both cant away from the coplanar state. Such a state does not arise in a simple model of frustrated magnetism on the kagome lattice. This type of canted ground state of the hexamer can tile the lattice, and therefore it is the building block of the classical ground state of the macroscopic system. There is a corresponding macroscopic degeneracy associated with the many ways in which this local hexamer ground state can tile the lattice. Each hexamer possesses a local moment; it is not yet clear whether the local magnetic moments from different hexamers cancel out due to the presence of zero modes.

\section{ACKNOWLEDGMENTS}

It is a pleasure to thank M. Ma for helpful discussions. D.X.Y. acknowledges support from Purdue University. This work was also supported by Research Corporation (Y.L.L. and E.W.C.).
${ }^{1}$ R. H. Fowler and G. S. Rushbrooke, Trans. Faraday Soc. 33, 1272 (1937).

${ }^{2}$ M. E. Fisher, Phys. Rev. 124, 1664 (1961).

${ }^{3}$ P. W. Kasteleyn, J. Math. Phys. 4, 287 (1963).

${ }^{4}$ P. W. Anderson, Mater. Res. Bull. 8, 153 (1973).

${ }^{5}$ D. S. Rokhsar and S. A. Kivelson, Phys. Rev. Lett. 61, 2376 (1988).

${ }^{6}$ R. Moessner and S. L. Sondhi, Phys. Rev. Lett. 86, 1881 (2001).

${ }^{7}$ A topological sector is defined as the following. Draw a line through the system, without touching any site. For a given topological sector, the number of dimers which cross that line is invariant mod 2 under local rearrangements of the dimer covering (see, e.g., Ref. 38).

${ }^{8}$ D. A. Huse, W. Krauth, R. Moessner, and S. L. Sondhi, Phys. Rev. Lett. 91, 167004 (2003).

${ }^{9}$ M. E. Fisher and J. Stephenson, Phys. Rev. 132, 1411 (1963).

${ }^{10}$ G. Misguich, D. Serban, and V. Pasquier, Phys. Rev. Lett. 89, 137202 (2002).

${ }^{11}$ P. Fendley, R. Moessner, and S. L. Sondhi, Phys. Rev. B 66, 214513 (2002).

${ }^{12}$ W. Krauth and R. Moessner, Phys. Rev. B 67, 064503 (2003).

${ }^{13}$ M. E. Fisher, J. Math. Phys. 7, 1776 (1966).

${ }^{14}$ R. Moessner and S. L. Sondhi, Phys. Rev. B 68, 054405 (2003).

${ }^{15}$ P. W. Anderson, Science 235, 1196 (1987).

${ }^{16}$ M. Gonzalez, F. Cervantes-Lee, and L. W. ter Haar, Mol. Cryst. Liq. Cryst. 233, 317 (1993).

${ }^{17}$ S. Maruti and L. W. ter Haar, J. Appl. Phys. 75, 5949 (1994).

${ }^{18}$ M. Mekata, M. Abdulla, T. Asano, H. Kikuchi, T. Goto, T. Morishita, and H. Hori, J. Magn. Magn. Mater. 177-181, 731 (1998).

${ }^{19}$ Y. L. Loh, D. X. Yao, and E. W. Carlson, Phys. Rev. B 77, 134402 (2008).

${ }^{20}$ D. X. Yao, Y. L. Loh, E. W. Carlson, and M. Ma, Phys. Rev. B 78, 024428 (2008).

${ }^{21}$ Kasteleyn's theorem may be generalized to allow complex phase factors in the weighted adjacency matrix: for a transition cycle passing through sites $1,2, \ldots, 2 n$, the phase factors must satisfy $\eta_{12} \eta_{34} \ldots \eta_{2 n-1,2 n}=-\eta_{23} \eta_{45} \ldots \eta_{2 n, 1}$. Complex phase factors provide a more elegant solution of the square lattice dimer model (Ref. 39). However, they do not help in the case of the kagome lattice (Ref. 23) or TKL; we have found that any orientation with the periodicity of the original lattice violates the generalized Kasteleyn theorem even if the phase factors are allowed to be arbitrary complex numbers.

${ }^{22}$ The expression for $S$ does not simplify appreciably when $F$, etc., are substituted in.

${ }^{23}$ F. Wang and F. Y. Wu, Phys. Rev. E 75, 040105(R) (2007).

${ }^{24}$ A. Läuchli and D. Poilblanc, Phys. Rev. Lett. 92, 236404 (2004).

${ }^{25}$ L. Balents, M. P. A. Fisher, and S. M. Girvin, Phys. Rev. B 65, 224412 (2002)

${ }^{26}$ The long-distance behavior can also be seen from the perspective of the quantum dimer model of Ref. 10, in that each monomer removes an Ising degree of freedom since it merges two hexagons. Therefore each monomer removes half of the configurations (Ref. 40).

${ }^{27}$ R. Moessner and S. L. Sondhi, Phys. Rev. B 68, 064411 (2003).

${ }^{28}$ A. J. Phares and F. J. Wunderlich, Nuovo Cimento Soc. Ital. Fis., B 101, 653 (1988).

${ }^{29}$ V. Elser, Phys. Rev. Lett. 62, 2405 (1989).

${ }^{30}$ V. Elser and C. Zeng, Phys. Rev. B 48, 13647 (1993).

${ }^{31}$ H. W. J. Blote and H. J. Hilborst, J. Phys. A 15, L631 (1982).

${ }^{32}$ D. A. Huse and V. Elser, Phys. Rev. Lett. 60, 2531 (1988).

${ }^{33}$ J. T. Chalker, P. C. W. Holdsworth, and E. F. Shender, Phys. Rev. Lett. 68, 855 (1992).

${ }^{34}$ R. Moessner and J. T. Chalker, Phys. Rev. B 58, 12049 (1998).

${ }^{35}$ J. N. Reimers and A. J. Berlinsky, Phys. Rev. B 48, 9539 (1993).

${ }^{36}$ I. Ritchey, P. Chandra, and P. Coleman, Phys. Rev. B 47, 15342 (1993).

${ }^{37}$ M. E. Zhitomirsky, Phys. Rev. B 78, 094423 (2008).

${ }^{38}$ S. Sachdev, Rev. Mod. Phys. 75, 913 (2003).

${ }^{39}$ T. T. Wu, J. Math. Phys. 3, 1265 (1962).

${ }^{40} \mathrm{G}$. Misguich (private communication). 